\title{
Sample Entropy Analysis of Hemodynamic Parameters in elderly with Orthostatic Intolerance
}

\author{
Marcos Hortelano $^{1}$, Richard B.Reilly ${ }^{2}$, Raquel Cervigón ${ }^{1}$ \\ ${ }^{1}$ Escuela Politcnica. UCLM. Camino del Pozuelo sn. 16071. Cuenca. Spain \\ ${ }^{2}$ School of Engineering and School of Medicine. Trinity College Dublin. Dublin 2, Republic of Ireland.
}

\begin{abstract}
This study focuses on examining the hemodynamic profile in older people with symptoms of orthostatic intolerance (OI) undergoing an active stand and to investigate if their dynamic cardiovascular profile during six-minute walk distance would be different to those of controls.

The database included a total of 65 participants, aged over 70 years of age (70.11 55.85 ), of whom $65 \%$ were females. The participants underwent a supine to stand orthostatic test with non-invasive beat to beat systolic $(S B P)$ and diastolic blood pressure $(D B P)$ and inter-beat interval $(R-R)$ monitoring. Sample Entropy (SampEn) method was calculated at each phase.

$R-R$ SampEn values showed statistically significant differences during Phase 2 and Phase 4 between symptomatic and asymptomatic OI participants. This study empathizes the importance of entropy analysis to extract information of cardiovascular signals. SampEn based measures might provide useful indicators of pathological changes in cardiac activity and be suitable for the early detection of autonomic cardiovascular dysfunction.
\end{abstract}

\section{Introduction}

Orthostatic intolerance (OI) is a clinical syndrome that is characterized by symptoms and loss of consciousness before impending syncope. It has been reported that is caused by orthostatic hypotension $(\mathrm{OH})$. Some previous studies suggest that the presence of $\mathrm{OH}$ may be a risk factor for falls and it increase risk of mortality and has been associated with a number of diseases including stroke, myocardial infarction and coronary artery disease $[1,2]$.

Cardiovascular signals are largely analysed using traditional time and frequency domain measures. The dynamic processes, especially under healthy conditions are evident in the complex fluctuations of physiologic output signals [3].

Several entropy measures have been defined in the literature. Although all of them are related to the same con- cept, the mathematical formulations vary among them. In previous studies have been applied to detect changes in the cardiovascular system in the pathological groups of chronic heart failure patients, patients after acute myocardial infarction (MI) and subjects during physical activity or adults with symptoms of OI [4-7].

This study focuses on examining the hemodynamic profile in older people with symptoms of OI undergoing an active stand and to investigate if their dynamic cardiovascular profile during a six-minute walk would be different to those of controls by calculating the sample entropy (SampEn).

\section{Material}

The database included a total of 65 participants, aged over 70 years of age $(70.11 \pm 5.85)$, of whom $65 \%$ were females. There was no significant differences in age and gender between symptomatic and asymptomatic OI participants, $44.6 \%(\mathrm{n}=29)$ had symptoms of OI and $55.4 \%$ $(\mathrm{n}=36)$ did not (Table 1).

Table 1. Database used to carry out our study.

\begin{tabular}{lccc}
\hline \hline & & Women & Men \\
\hline OI & Participants & 23 & 13 \\
& Age & $69.65 \pm 5.26$ & $71.77 \pm 6.47$ \\
\hline non-OI & Participants & 19 & 10 \\
& Age & $70.63 \pm 5.34$ & $68.00 \pm 7.21$ \\
\hline \hline
\end{tabular}

The participants were evaluated in the Technology Research for Independent Living Clinic (TRIL) at St Jame's hospital in Dublin [8]. To carry out this study, the approval of the Local Research Ethics Commitee (SJH/AMNCH Research Ethics Commitee approval reference number 13/06/2007) was necessary. Every participant got a minimum score of 23 in a Minimental state examination (MMSE), which is the threshold of insanity detection in the Irish framework [9]. All subjects consented before being included in this study. None of the participants suffered 
from Parkinson, diabetes mellitus, acute chronic renal failure, deficiency in B12 vitamin or a pacemaker. None of the participants took diuretics, ace inhibitor or angitotension II. The measurement of the BP wave was carried out using the Finapres method (Finger Arterial Pressure).

\section{Methods}

\section{1. $\quad$ Procedure}

To carry out this study, the 6 minutes walking distance test (6MWD) was applied. This test consist basically on measuring the distance which a person can walk in 6 minutes and it must be done along a long, flat an straight 30 meter long corridor. The 6MWD test is divided into clearly five identified and differentiated phases: a first phase of rest with a duration of 3 minutes, Phase 1 (pre-exercise). During the Phase 2 our participants begin the walk (starting of exercise). The Phase 3, with a duration of 6 minutes, in which participants are doing exercise (active phase). The Phase 4 which started when our participants ended the walk until they have recovered (recovery phase) and the Phase 5 at rest with a duration of 3 minutes (post-exercise). Due to this fact, we are going to work with a total of five phases (Phase 1, Phase 2, Phase 3, Phase 4, Phase 5). The same number of intervals was extracted in each of the five phases when the signal was stabilized ( $n=180$ samples) (Figure 1).

The following hemodynamic measurements beat by beat were registered:

- SBP: as the maximum blood pressure in the systole (mmHg).

- DBP: as the minimum value of pressure in the diastole (mmHg).

- R-R: R-R interval in miliseconds (ms).

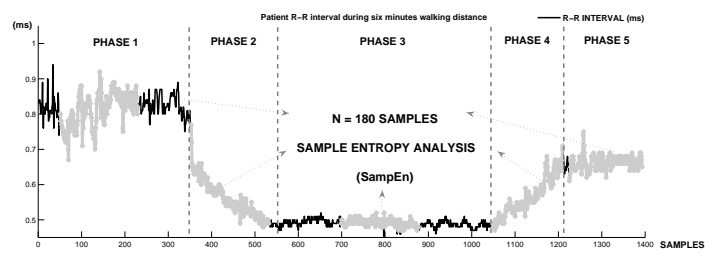

Figure 1. Patient R-R during six minutes walking distance.

\subsection{Sample Entropy}

In this study, SampEn [10], was chosen as a characterization of activation patterns on the OI. This is a similar but less biased measure than the approximate entropy (ApEn) family parameters, introduced by Pincus [11] to quantify the regularity of finite length time series.

SampEn is precisely equal to the negative of the natural logarithm of the ratio between the probability of oc- currence of sequences close to each other (according to a threshold value $r$ to measure the distance between sequences) for $m$ consecutive data points within a chosen distance $m\left(U_{m}(r)\right)$, and the probability of occurrence of sequences close to each other when one more point is added to each sequence [12]. Larger SampEn values indicate greater independence, less predictability, hence greater complexity in the data:

$$
\operatorname{SampEn}(m, r, N)=-\ln \frac{U^{m+1}(r)}{U^{m}(r)}
$$

SampEn is estimated using the widely established parameter values of $m=2$ and $r=0.25 \delta$, where $\delta$ represents the standard deviation of the original data sequence [13]

\subsection{Statistical analysis}

An analysis of variance (ANOVA) was employed to test differences between phases and between groups for each hemodynamic parameter. Comparisons of continuous variables between the two groups were performed by Student's unpaired t-test or Mann-Whitney U test, where appropriate. Analysis of variance with repeated measurements ANOVA and with the Student-Newman-Keuls test were used to study the effect of one or more factors when at least one of them is a within-subjects factor or related measurements. When a significant overall effect was detected, Scheffe's F-test was used for comparison of the mean values for the two groups. The results were regarded as statistically significant for $\mathrm{p}<0.05$.

\section{Results}

In a first analysis, differences in the regularity hemodynamic measurements of SBP and DBP between symptomatic OI group and asymptomatic OI group were studied. The most significant results were observed in SBP SampEn in the post-exercise, showing statistically differences with statistical significance $(\mathrm{p}=0.023)$ between symptomatic OI group $(1.05 \pm 0.22)$ and asymptomatic group (0.89 \pm 0.35$)$ (Figure 2).

Nevertheless, the major differences were found in the hemodynamic measurement of R-R, showing statistically significant differences in Phase $2(\mathrm{p}=0.015)$ and Phase 4 $(\mathrm{p}=0.024)$ (Table 2$)$. In this analysis, the symptomatic OI group illustrated higher entropy SampEn values compared with the asymptomatic group (Figure 3). 


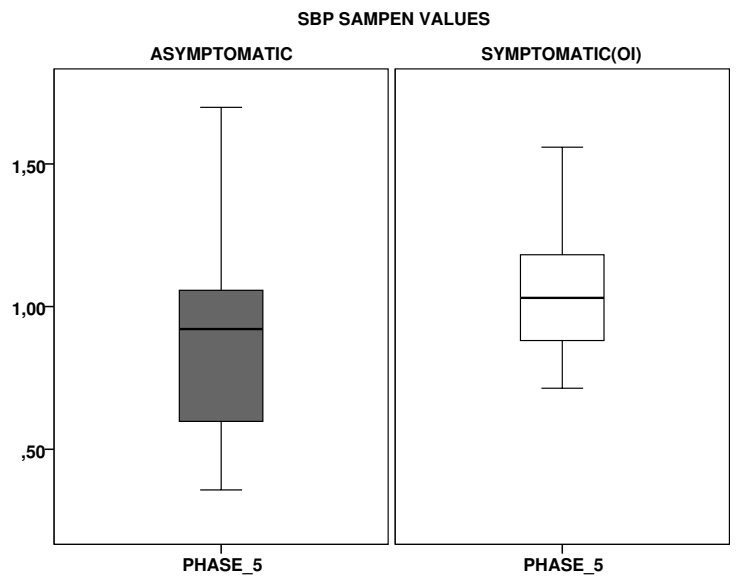

Figure 2. SBP SampEn values in Phase 5.

Table 2. SampEn results in Phase 2 and Phase 4.

\begin{tabular}{ccccc}
\hline \hline SampEn & Phase & OI & non-OI & (p) \\
\hline R-R & Phase 2 & $0.75 \pm 0.46$ & $0.52 \pm 0.35$ & 0.015 \\
\hline R-R & Phase 4 & $0.71 \pm 0.40$ & $0.50 \pm 0.38$ & 0.024 \\
\hline \hline
\end{tabular}

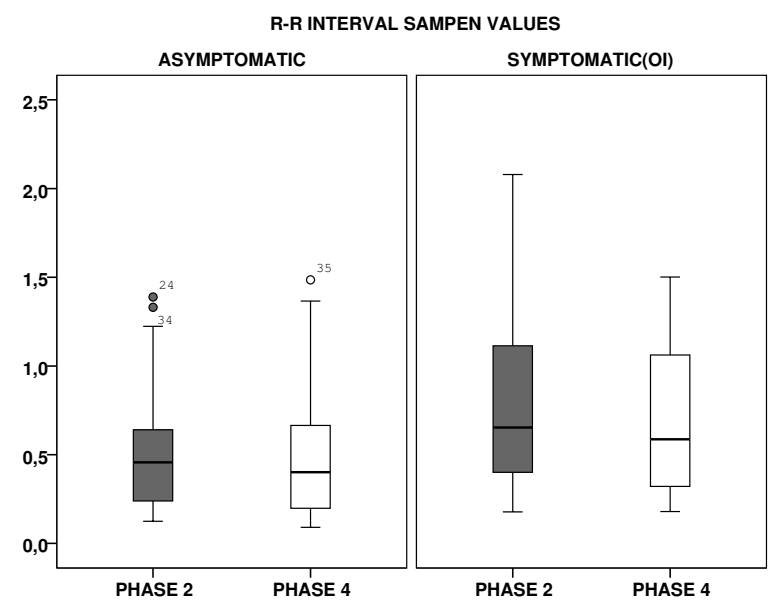

Figure 3. RR SampEn values in Phase 2 and Phase 4.

When the distinction between men and women was made, we were able to observe greater differences in the male group (Figure 4). SampEn analysis showed statistically significant differences in starting of exercise phase $(\mathrm{p}=0.021)$ and recovery phase $(\mathrm{p}=0.037)$ between symptomatic OI men group and asymptomatic men group (Table 3).

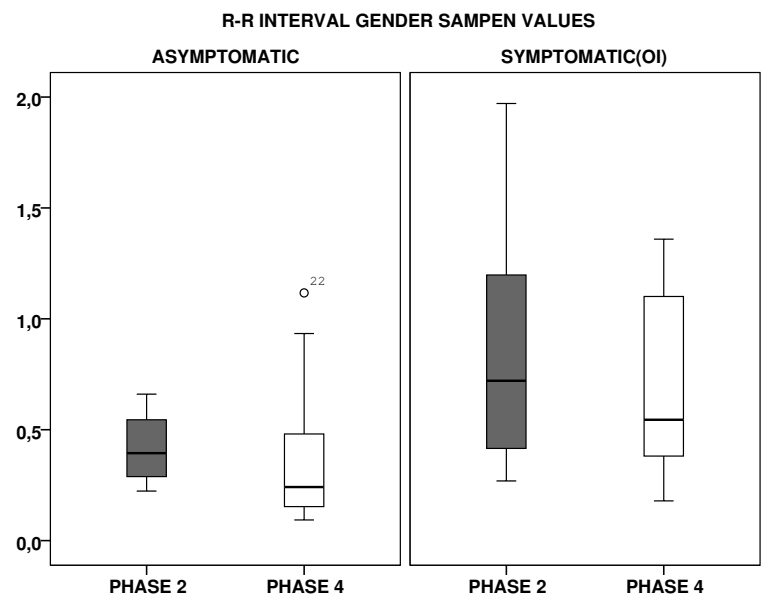

Figure 4. Gender differences in Phase 2 and Phase 4.

Table 3. Gender differences in Phase 2 and Phase 4.

\begin{tabular}{ccccc}
\hline \hline SampEn & Phase & Men OI & Men non-OI & $(\mathrm{p})$ \\
\hline R-R & Phase 2 & $0.83 \pm 0.50$ & $0.42 \pm 0.16$ & 0.021 \\
\hline R-R & Phase 4 & $0.75 \pm 0.43$ & $0.39 \pm 0.35$ & 0.037 \\
\hline \hline
\end{tabular}

\section{Conclusion}

This study analyze by non linear methods differences in the response of symptomatic OI under 6MWD test. During starting of exercise and recovery phase the SampEn $\mathrm{R}-\mathrm{R}$ interval decreased in asymptomatic OI group compared with the symptomatic OI group (Figure 3). Moreover, these results are coherent with previous studies where an abnormal complex cardiac functioning in symptomatic OI patients were observed [14]. In addition, in both groups we were able to observe a decrease in SampEn values in Phase 2 and Phase 4 if we compared with pre-exercise and post-exercise stages, being a higher difference in the asymptomatic group. In other articles, similar results were shown, where the head-up tilt test resulted in a significant reduction in SampEn of R-R interval, these measures of entropy are suitable to detect changes in neural outflow to the heart and decoupling of repolarization variability from heart rate variability elicited by orthostatic stress [15].

On the other hand, the symptomatic OI group showed higher SampEn values in all stages, but these differences between both groups did not reach statistical significance. This different behaviour in the elderly may be is explained with physiological aging, which is associated with a reduction of autonomic control on the cardiac rhythm, demonstrated by reduction in all the time domain HRV indices $[4,7,16]$. Once we analyzed gender difference, we observed that the group of men illustrated a higher pathological differences, showing greater SampEn values in start- 
ing of exercise and recovery stages. Previous articles illustrated changes in the multiscale time irreversibility analysis within the symptomatic OI men group, showing a decrease in the irreversibility indices in the recovery stages [14]. Similarly, other studies found that neuroendocrine control of blood pressure in healthy subjects was significantly higher in older men compared with the younger men and women of both age groups, suggesting a divergent age-related activation of the sympathetic nervous system between genders at baseline as well as during a sustained orthostatic challenge [17]. Nevertheless, other studies suggests that aging rather than sex needs to be taken into account when interpreting R-R and BP changes during prolonged orthostatic test such as head-up tilt test performance [18].

This study is focused on therapies and interventions that potentially might enhance hemodynamic response. As conclusion, this study empathizes the importance of entropy analysis to extract information of cardiovascular signals with non-linear methods, results provide useful indicates of pathological changes in cardiac activity and be suitable for the early detection of autonomic cardiovascular dysfunction.

\section{Acknowledgements}

This work was supported by the Castilla-La Mancha Research Scheme (PPII- 20 14-024-P).

\section{References}

[1] Gangavati A, Hajjar I, Quach L, Jones RN, Kiely DK, Gagnon P, Lipsitz LA. Hypertension, orthostatic hypotension, and the risk of falls in a community-dwelling elderly population: The maintenance of balance, independent living, intellect, and zest in the elderly of boston study. Journal of the American Geriatrics Society 2011;59(3):383-389.

[2] Heitterachi E, Lord SR, Meyerkort P, McCloskey I, Fitzpatrick R. Blood pressure changes on upright tilting predict falls in older people. Age and ageing 2002;31(3):181-186.

[3] Droguett VSL, da Cruz Santos A, de Medeiros CE, Marques DP, do Nascimento LS, do Socorro Brasileiro-Santos M. Cardiac autonomic modulation in healthy elderly after different intensities of dynamic exercise. Clinical interventions in aging 2015;10:203.

[4] Hortelano M, Reilly RB, Cogan L, Cervigón R. Heart Rate Regularity Changes in Older People with Orthostatic Intolerance. Cham: Springer International Publishing. ISBN 978-3-319-16483-0, 2015; 337-346.

[5] Casali KR, Casali AG, Montano $\mathrm{N}$, Irigoyen $\mathrm{MC}$, Macagnan F, Guzzetti S, Porta A. Multiple testing strategy for the detection of temporal irreversibility in stationary time series. Physical Review E 2008;77(6):066204.

[6] Porta A, Casali KR, Casali AG, Gnecchi-Ruscone T, Tobaldini E, Montano N, Lange S, Geue D, Cysarz D, Van Leeuwen P. Temporal asymmetries of short-term heart period variability are linked to autonomic regulation. American Journal of Physiology Regulatory Integrative and Comparative Physiology 2008;295(2):R550-R557.

[7] Hortelano M, Reilly RB, Cervigón R. Multiscale entropy analysis of heart rate regularity changes in older people with orthostatic intolerance. In XIV Mediterranean Conference on Medical and Biological Engineering and Computing 2016. Springer, 2016; 62-66.

[8] Kenny RA, Dishongh T, Newell F, Ni Scanail C. Research to reduce falls in older people: the tril centre. Geriatr Med 2009;39:326-327.

[9] Cullen B, Fahy S, Cunningham CJ, Coen RF, Bruce I, Greene E, Coakley D, Walsh JB, Lawlor BA. Screening for dementia in an irish community sample using mmse: a comparison of norm-adjusted versus fixed cut-points. International journal of geriatric psychiatry 2005;20(4):371376.

[10] Richman JS, Moorman JR. Physiological time-series analysis using approximate entropy and sample entropy. American Journal of Physiology Heart and Circulatory Physiology 2000;278(6):H2039-H2049.

[11] Pincus S. Approximate entropy (apen) as a complexity measure. Chaos An Interdisciplinary Journal of Nonlinear Science 1995;5(1):110-117.

[12] Cervigón R, Moreno J, Reilly R, Millet J, Pérez-Villacastín $\mathrm{J}$, Castells F. Entropy measurements in paroxysmal and persistent atrial fibrillation. Physiological measurement 2010; 31(7):1011.

[13] Pincus SM. Approximate entropy as a measure of system complexity. Proceedings of the National Academy of Sciences 1991;88(6):2297-2301.

[14] Hortelano M, Reilly RB, Cervigón R. Multiscale time irreversibility to predict orthostatic intolerance in older people ;.

[15] Baumert M, Czippelova B, Ganesan A, Schmidt M, Zaunseder S, Javorka M. Entropy analysis of rr and qt interval variability during orthostatic and mental stress in healthy subjects. Entropy 2014;16(12):6384-6393.

[16] Laitinen T, Niskanen L, Geelen G, Länsimies E, Hartikainen J. Age dependency of cardiovascular autonomic responses to head-up tilt in healthy subjects. Journal of Applied Physiology 2004;96(6):2333-2340.

[17] Geelen G, Laitinen T, Hartikainen J, Länsimies E, Bergström K, Niskanen L. Gender influence on vasoactive hormones at rest and during a 70 head-up tilt in healthy humans. Journal of Applied Physiology 2002;92(4):14011408.

[18] Ndayisaba JP, Fanciulli A, Granata R, Duerr S, Hintringer F, Goebel G, Krismer F, Wenning GK. Sex and age effects on cardiovascular autonomic function in healthy adults. Clinical Autonomic Research 2015;25(5):317-326.

Address for correspondence:

Marcos Hortelano Rubio

Escuela Politecnica. Camino del Pozuelo sn, Cuenca. Spain

Marcos.Hortelano@uclm.es 